\title{
Patients' Acceptability of Different Fentanyl Products for Breakthrough Cancer Pain
}

Sir - Transmucosal administration of fentanyl products are increasingly used for breakthrough pain [1]. As little is known regarding the patients' view about their acceptability, 75 consecutive advanced cancer patients were surveyed to assess the acceptability of these delivery systems. Patients receiving available fentanyl products were asked medications, as patients' education and compliance are the most important factors for appropriate use of these agents [4]. Such preliminary data on a sample of patients who accepted to be interviewed should be confirmed by studies with specific sampling and randomisation in different settings.

Table 1

ROOs ( $n^{\circ}$ patients) and mean values (SD) of the following items: easy of administration, modality of administration, palatability, satisfaction, overall impression, and time for pain relief (minutes).

\begin{tabular}{lllllll}
\hline & Easy & Modality & Palatability & Satisfaction & Overall impression & Time for pain relief \\
\hline OTFC (6) & $1.7(0.5)$ & $1.5(0.5)$ & $2.0(0.6)$ & $1.5(0.8)$ & $1.5(0.5)$ & $33.1(43.5)$ \\
FBT (19) & $2.0(0.6)$ & $2.0(0.7)$ & $1.8(0.7)$ & $1.8(0.8)$ & $1.9(0.7)$ & $15.9(7.3)$ \\
SLF (37) & $2.1(0.5)$ & $2.2(0.4)$ & $1.8(0.4)$ & $2.0(0.6)$ & $2.1(0.5)$ & $16.7(12.5)$ \\
INFS (7) & $2.1(0.4)$ & $2.1(0.4)$ & $2.0(0.6)$ & $2.0(0.8)$ & $2.0(0.8)$ & $15.7(13.7)$ \\
PFEN (11) & $2.4(0.5)$ & $2.2(0.4)$ & $1.8(0.4)$ & $1.9(0.7)$ & $2.1(0.5)$ & $15.0(13.7)$ \\
$P$ & 0.120 & $0.043^{*}$ & 0.870 & 0.595 & 0.178 & $0.015 \S$ \\
\hline
\end{tabular}

", Oral transmucosal fentanyl was significantly considered to be more problematic.

about ease of administration, modality of administration, palatability, satisfaction and global impression. For patients who were receiving nasal products, palatability was substituted with sense of smell. Responses were rated on a 0-3 Likert scale. Rapid onset opioids (ROOs) were globally assessed 80 times (five patients received more ROOs).

Oral transmucosal fentanyl was significantly considered to be more problematic for modality of administration and late pain relief $(P=0.043$ and 0.015 , respectively) (Table 1 ), possibly due to the need for patient collaboration, not always possible in patients with weakness. Other fentanyl products were well accepted by patients, in terms of ease and modality of administration, palatability and overall impression. Data regarding these practical aspects of administering breakthrough pain medications have been rarely examined. In a Canadian survey, patients expressed a willingness to try transmucosal products $(80 \%)$ or nasal products $(59 \%)$ [2]. In a recent survey, fentanyl products were given as placebo. The usual rescue analgesic and sublingual fentanyl were rated better than fentanyl buccal tablet and intranasal fentanyl for accessibility. It was unclear what the usual rescue analgesic was [3]. The second generation of ROOs seems to have favourable characteristics for some practical issues. This is a fundamental aspect regarding the use of these
S. Mercadante*, A. Caruselli $\dagger$, M.R. Meli + , G. Bellavia $\dagger$, A. Tuttolomondo $\$$, A. Casuccio

* Pain Relief and Palliative Care Unit, La Maddalena Cancer Center, Palermo, Italy

† SAMO, Home Care Program, Italy

${ }^{\ddagger}$ Faculty of Pharmacy, University of Palermo, Palermo, Italy

${ }^{\S}$ Dipartimento Biomedico di Medicina Interna e Specialistica (DIBIMiS), Università degli Studi di Palermo, Palermo, Italy

"Department of Sciences for Health Promotion and Mother-Child Care "G. D'Alessandro", University of Palermo, Palermo, Italy

\section{References}

[1] Mercadante S. Pharmacotherapy for breakthrough cancer pain. Drugs 2012;72:181-190.

[2] Bedard G, Hawley P, Zhang L, et al. A survey of Canadian cancer patients' perspectives on the characteristics and treatment of breakthrough pain. Support Care Cancer 2013;21:2557-2563.

[3] England R, Maddocks M, Manderson C, ZadoraChrzastowska S, Wilcock A. How practical are transmucosal fentanyl products for breakthrough cancer pain? A survey of user opinion. Br Med J Support Palliat Care 2011;1:349-351.

[4] Webber K, Davies AN, Cowie MR. Breakthrough pain: a qualitative study involving patients with advanced cancer. Support Care Cancer 2011;19:2041-2046.

http://dx.doi.org/10.1016/j.clon.2014.09.003

(C) 2014 The Royal College of Radiologists. Published by Elsevier Ltd. All rights reserved 